\title{
Plasmocitoma de base craneal con inestabilidad cráneo-cervical
}

\author{
N. Samprón; M. Arrazola y E. Urculo
}

Servicio de Neurocirugía. Hospital Donostia. San Sebastián. España.

\section{Resumen}

Introducción. La inestabilidad cráneo-cervical constituye, en ocasiones, el principal problema neuroquirúrgico en la patología tumoral de la base craneal posterior. Presentamos un caso clínico en el que un plasmocitoma solitario originó inestabilidad cráneocervical. Durante la cirugía de estabilización, se lesionó la arteria vertebral. Revisamos la anatomía quirúrgica desde el punto de vista de la prevención de las complicaciones vasculares.

Caso clínico. Mujer de 66 años diagnosticada de plasmocitoma solitario de base craneal, tratada con radio y quimioterapia con remisión completa, que presenta tetraparesia y disfagia. Tras el diagnóstico de inestabilidad cráneo-cervical, se indica estabilización mediante instrumentación occipito-cervical. Es intervenida bajo tracción craneal con atornillado C1-C2 según técnica de Magerl y extensión occipital. Durante la cirugía se lesionó la arteria vertebral derecha sin repercusión clínica. Dos años más tarde, la paciente es capaz de llevar una vida independiente.

Conclusiones. La instrumentación cráneo-cervical con tornillos transarticulares $\mathrm{C} 1-\mathrm{C} 2$, como parte del sistema de fijación C0-C1-C2, parece eficaz para corregir la inestabilidad en lesiones osteolíticas, a expensas de un riesgo considerable de lesión de la arteria vertebral, especialmente en presencia de algunas variaciones anatómicas.

PALABRAS CLAVE: Base craneal. Plasmocitoma solitario. Inestabilidad cráneo-cervical. Arteria vertebral. Instrumentación.

Skull base plasmacytoma with craniocervical instability

Summary

Introduction. Cranio-cervical instability is, in some

Recibido: 20-01-08. Aceptado: 10-07-08 cases, the main surgical concern in posterior skull base tumors. We report on a case in which a solitary plasmacytoma of the skull base presented with cranio-cervical instability. Vertebral artery was injured during surgery. The surgical anatomy is reviewed, with emphasis in vascular complications avoidance.

Case report. A 66 year-old woman was diagnosed of a cranial base solitary plasmacytoma and treated with radio and chemotherapy with complete remission. After receiving that treatment, she presented with tetraparesis and a cranio-cervical instability was diagnosed. She was operated on, under cranial traction, of posterior occipito-cervical instrumentation with $\mathrm{C} 1$ to C2 transarticular Magerl screws. The right vertebral artery was injured during surgery without additional neurological deficit. Two years after the operation she remains independent for daily activities.

Conclusions. Transarticular screws at the $\mathrm{C} 1$ to $\mathrm{C2}$ level of the cervical spine may provide rigid fixation in posterior cranio-cervical instrumentation for osteolytic lesions, but there is a risk of injury to the vertebral artery, specially when some variations in the surgical anatomy exist.

KEY WORDS: Skull base. Solitary plasmacytoma. Craniocervical instability. Screw. Vertebral artery.

Introducción

La estabilidad cráneo-cervical depende de la integridad de la base craneal posterior, el atlas, el axis, el complejo articular, ligamentario y muscular ${ }^{13}$. Diversos procesos de origen inflamatorio, neoplásico, traumático, malformativo o degenerativo que afectan a la región cráneo-cervical pueden generar inestabilidad ${ }^{10}$. Esta se expresa clínicamente en un espectro que va desde las vagas molestias cervicales hasta la muerte súbita ${ }^{15}$.

El tratamiento de la inestabilidad cráneo-cervical suele requerir un procedimiento de descompresión y uno de fijación $^{12}$. La estabilización puede conseguirse con una ortesis externa con o sin injertos óseos, con instrumentación 


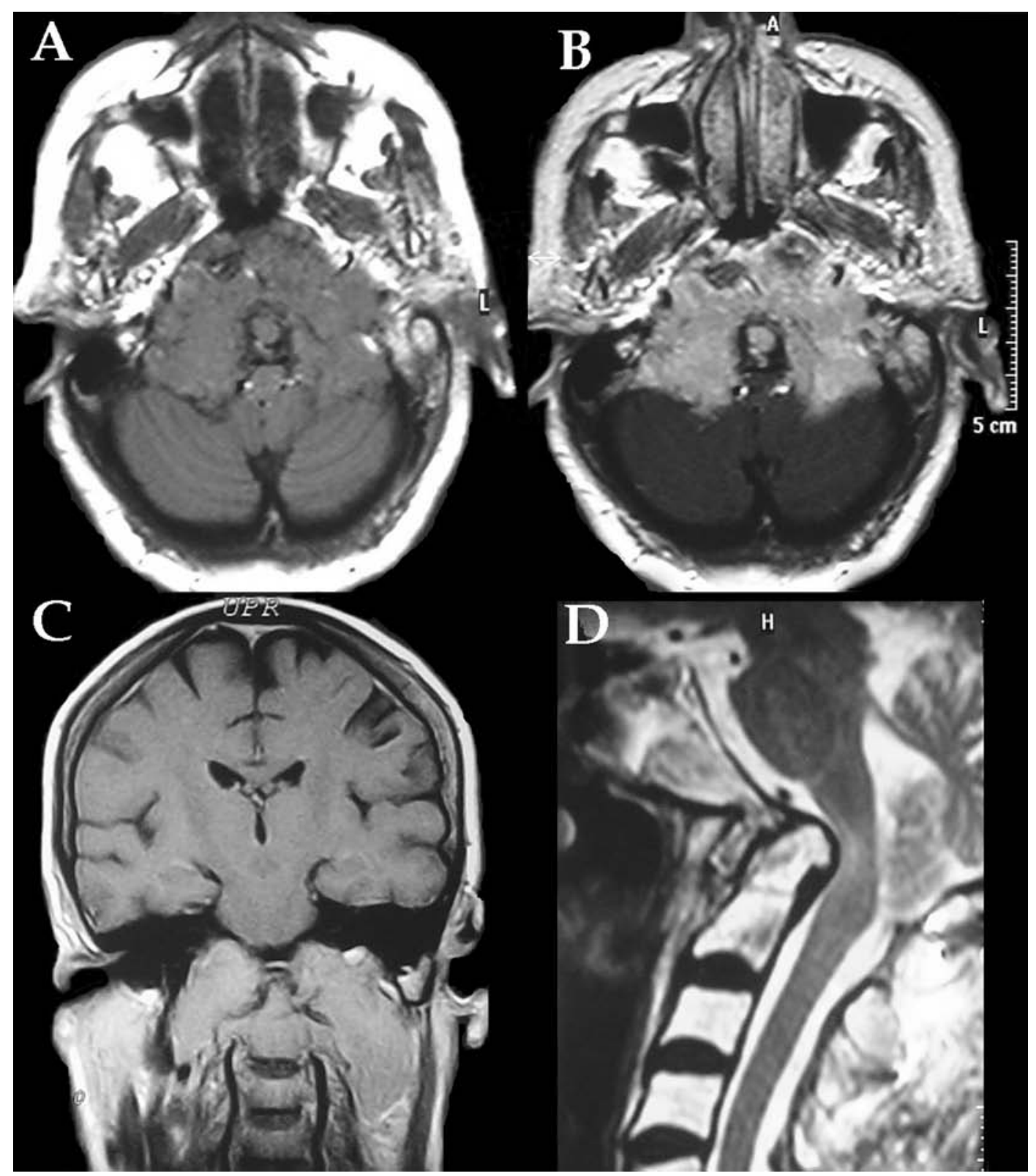

Figura 1. Resonancia magnética preoperatoria. (A y B) corte axial en T1 sin y con contraste a nivel de los cóndilos occipitales. Se aprecia una masa infiltrante que ha invadido ambos cóndilos, parte del clivus y de ambos peñascos con captación heterogénea del contraste. (C) proyección coronal en T1. El tumor destruye ambas masas laterales de C1, se extiende al área paravertebral, incorpora las arterias vertebrales y respeta al axis. (D) Proyección sagital en T2 que muestra una lesión hiperintensa en el bulbo raquídeo con impresión basilar.

cráneo-cervical, o con una combinación de estos procedimientos. En otras ocasiones, un único procedimiento es suficiente, sea descompresivo o de estabilización ${ }^{10,22,23}$.

En el presente caso, la paciente presenta un plasmocitoma solitario -versión localizada del mieloma múltiplecentrado en la región condilar del hueso occipital, que crece a partir de la médula ósea e induce la destrucción osteolítica de las estructuras adyacentes ${ }^{11,20}$.

\section{Caso clínico}

Mujer de 66 años que comienza con dolor cervical. Dos años más tarde se agregan parestesias en el miembro superior izquierdo y cefalea. Se indica una RM que revela una masa cráneo-cervical (Figura 1) que es biopsiada a través del cavum. El estudio anatomopatológico se corresponde con un tumor de células plasmáticas. El estudio de 


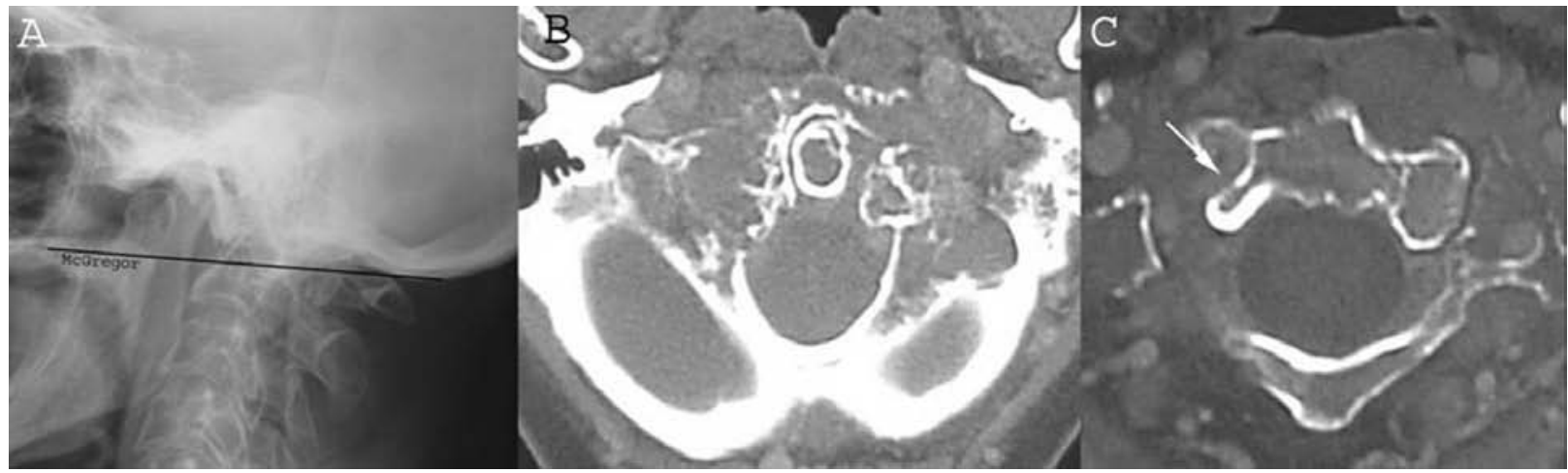

Figura 2. Estudio preoperatorio. (A) RX en proyección lateral en la que destaca una invaginación basilar valorada con la línea de referencia de McGregor. (B) TAC en projección axial a nivel de los cóndilos occipitales que pone de manifiesto osteólisis y la apófisis odontoides dentro del foramen mágnum. (C) TAC en proyección axial a nivel de cuerpo del axis en el que se puede intuir un surco de arteria vertebral profundo del lado derecho (flecha).

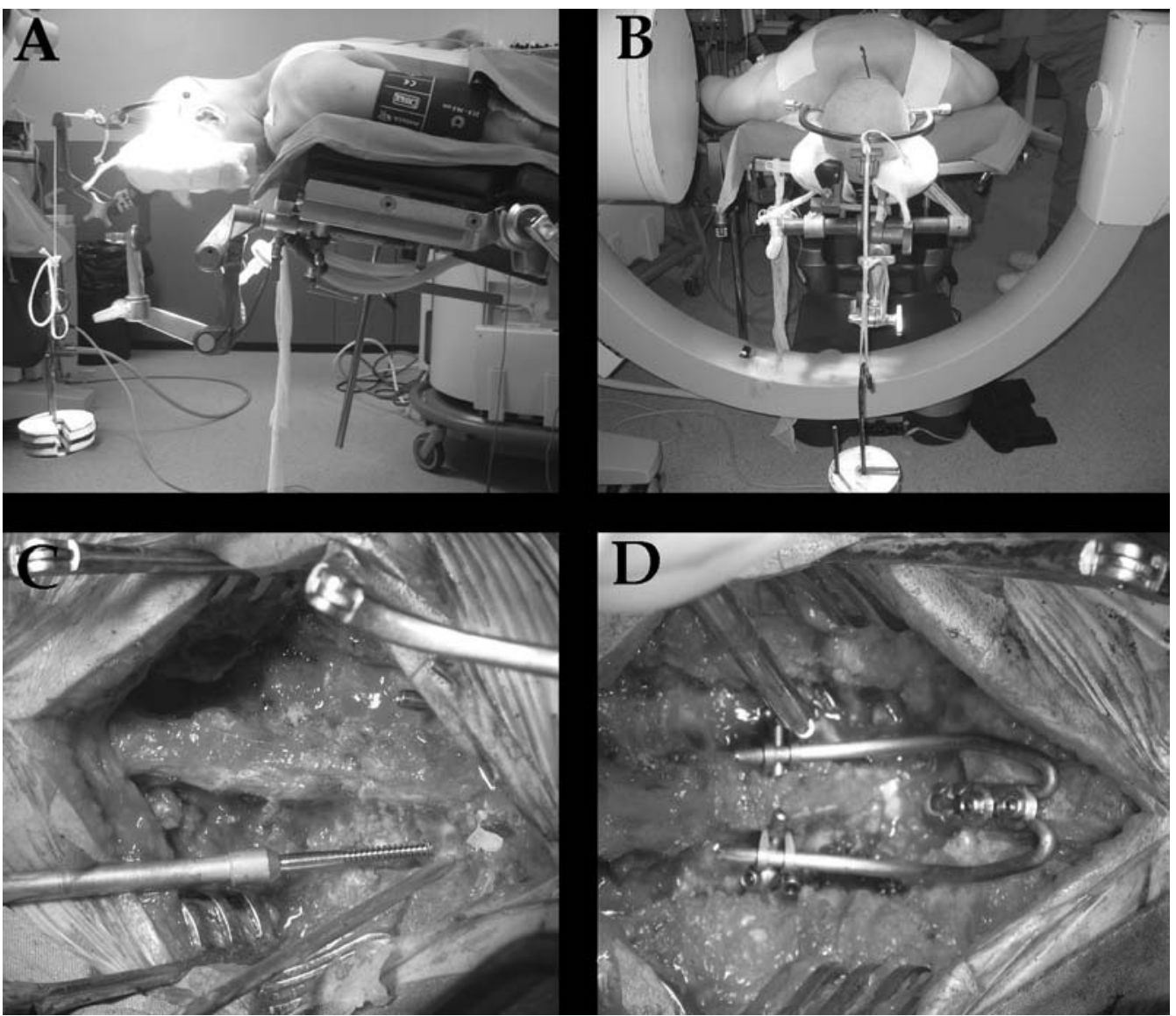

Figura 3. Imágenes intraoperatorias. $\left(\begin{array}{lll}A & y & B\end{array}\right)$ paciente en posición de concorde con tracción craneal de $3 \mathrm{Kg}$. en flexión. (C) introducción del tornillo $\mathrm{C1} 1-\mathrm{C} 2$ derecho, el izquierdo ya ha sido colocado. (D) Aspecto final. Nótese cómo la barra occipitocervical no permite una craniectomía más extensa. extensión tumoral no muestra otras alteraciones. Se trata con quimioterapia y radioterapia (4 ciclos de Vincristina-Adriamicina-Dexametasona $+46,8$ Gy). Tras recibir este tratamiento la remisión tumoral es completa, pero la paciente comienza con tetraparesia, vértigos y disfagia. Se diagnostica inestabilidad cráneo-cervical con lesión del tronco cerebral y se indica fijación occipito-cervical (Figuras 1 y 2). La cirugía se realizó en posición de con- corde, con tracción craneal de $3 \mathrm{Kg}$ en ligera flexión que permitió una modesta reducción de la impresión basilar (Figura 3 A y B). Se realizó un pequeña craniectomía suboccipital con extirpación del arco posterior del atlas. Se instrumentó con el sistema Olerud ${ }^{\circledR}$ (Anatomica, Gothenburg, Sweden) y tornillos transarticulares C1-C2 según la técnica de Magerl ${ }^{7,14}$ (Figura $3 \mathrm{C}$ y D). Al colocar el tornillo transarticular derecho (Figura $3 \mathrm{C}$ ), se lesionó la 
arteria vertebral, consiguiendo la hemostasia con la introducción completa del tornillo. No hubo incremento de los déficit neurológicos en el postoperatorio inmediato; sin embargo, este se complicó con una hemorragia digestiva alta y con aspergilosis pulmonar. La paciente respondió favorablemente al tratamiento médico de estas complicaciones y fue dada de alta a su domicilio. Un mes más tarde ingresa por disfasia. Se diagnostica de dos lesiones cerebrales que fueron biopsiadas. El diagnóstico de estas lesiones fue aspergiloma cerebral. La paciente evolucionó favorablemente con tratamiento médico y tras dos años de seguimiento lleva una vida independiente con un síndrome piramidal residual.

\section{Discusión}

Este caso sitúa a la inestabilidad cráneo-cervical como principal problema neuroquirúrgico en la patología tumoral de la base craneal posterior. Se trata de un tumor radio y quimiosensible, potencialmente curable ${ }^{20}$. El plasmocitoma ocasionó la destrucción osteolítica de los cóndilos occipitales y parte de las masas laterales del atlas, principales estructuras de estabilización de la charnela cráneo-cervical. Adicionalmente, el tumor engloba ambas arterias vertebrales y respeta las masas laterales del axis.

Se ha sugerido que la instrumentación con tornillos transarticulares C1-C2, según la técnica de Magerl ${ }^{5,14}$, es la técnica más eficaz desde el punto de vista biomecánico para la estabilización de las dos primeras vértebras, porque limita los movimientos de rotación, disminuyendo la posibilidad de pseudoartrosis y obviando la necesidad de ortesis postoperatoria. Además, esta técnica no depende de la integridad de los elementos posteriores. Por estas razones, actualmente se la considera el gold standard o patrón de referencia en la instrumentación de las dos primeras vértebras ${ }^{2,10}$.Otra ventaja de esta técnica cuando se utiliza en la fijación cráneo-cervical es que no requiere la instrumentación de múltiples niveles, aunque algunos cirujanos prefieren reforzar esta osteosíntesis incluyendo algún otro elemento ${ }^{8}$.

Sin embargo, en comparación con otras técnicas, el atornillado C1-C2 de Magerl puede asociarse a un mayor riesgo de lesión de la arteria vertebral. Una de las razones es que existe una enorme variabilidad anatómica en la pars interarticularis del axis, en las masas laterales y especialmente en el surco que la arteria vertebral labra sobre este ${ }^{1,9,10,14}$. Por ejemplo, la profundidad del surco de la arteria vertebral en la masa lateral de $\mathrm{C} 2$ es en promedio de 4,2 $\mathrm{mm}$ pero dentro de un amplio rango que va de 1 a $9,4 \mathrm{~mm}^{1}$. El trayecto de la arteria vertebral a este nivel es, por lo tanto, variable en forma, tamaño, localización y simetría. Estudios radiológicos y en cadáveres sugieren que existe una anatomía desfavorable para la colocación de estos tornillos, por lo menos en un lado, en el 18-23\% de los casos debido al trayecto de la arteria vertebral ${ }^{1,10,14,18}$. En concreto, se ha estimado el riesgo de lesión de la arteria vertebral durante la inserción de los tornillos trasarticulares C1-C2 en $4-8 \%{ }^{10,14,24,25}$. Sorprendentemente, se ha informado que sólo el 3,7\% de los pacientes con lesión del la arteria vertebral sufrieron repercusión clínica, por lo que el riesgo de déficit neurológico por daño en la arteria vertebral en esta técnica sería del 0,2\%14,25. El riesgo de muerte por lesión bilateral de la arteria vertebral se estima, a su vez, en $1,9 \%{ }^{25}$. La arteria vertebral izquierda es dominante en un $36 \%$ de los casos, la dominancia es compartida en el $40,8 \%$ de los $\operatorname{casos}^{14}$. Estos datos anatómicos sumado al hecho de que la lesión del vaso puede no asociarse a su oclusión definitiva pueden justificar esta falta de expresión clínica. La instrumentación transarticular $\mathrm{C} 1-\mathrm{C} 2$ está contraindicada ante la presencia de marcada cifosis, obesidad mórbida, tórax en tonel y ante la presencia de una anatomía desfavorable que es posible conocer de antemano con los estudios radiológicos ${ }^{9,10}$. La reducción de la subluxación, mediante tracción craneal, previo a la instrumentación, disminuye el riesgo de malposición de los tornillos y de complicaciones ${ }^{10}$. La instrumentación guiada por imágenes (navegación raquídea) puede aumentar a precisión en la trayectoria, limitar la exposición quirúrgica necesaria y disminuir el riesgo de complicaciones ${ }^{2,7,10}$. Sin embargo, si se ha conseguido una reducción significativa en quirófano, la precisión de esta técnica puede disminuir, de forma análoga al brain shift de la navegación craneal.

En el presente caso, vista la situación anatómica concreta (profundos surcos de la arteria vertebral en C2), otras formas de instrumentación podrían haber ofrecido mayor seguridad, disminuyendo el riesgo de lesión vascular. Una vez producida la lesión, se suele controlar el sangrado con la colocación definitiva del tornillo y se contraindica la colocación del tornillo del lado opuesto ${ }^{2}$. Por otro lado, la inclusión de áreas afectadas por el tumor dentro de la instrumentación (masas laterales de C1), no parece haber comprometido la estabilidad biomecánica.

Una alternativa que tampoco requiere la integridad de los elementos posteriores es la fijación con tornillos independientes para las masas laterales de $\mathrm{C} 1$ y $\mathrm{C} 2$ (técnica de Goel $)^{3,4,6,10,14}$ que reduce el riesgo de lesión de la arteria vertebral con una estabilidad biomecánica incluso superior $^{10}$, sin embargo no existen datos sobre el resultado de esta técnica a largo plazo. Se ha sugerido que esta técnica podría reemplazar al gold standard por ser más segura y más eficaz ${ }^{10}$.

Si los elementos posteriores de C1 y C2 están intactos y no se planea extirparlos, existen alternativas bien conocidas a la técnica de Margerl, que parecen más seguras aunque menos eficaces (con menor estabilidad biomecánica) como la instrumentación con gan- 


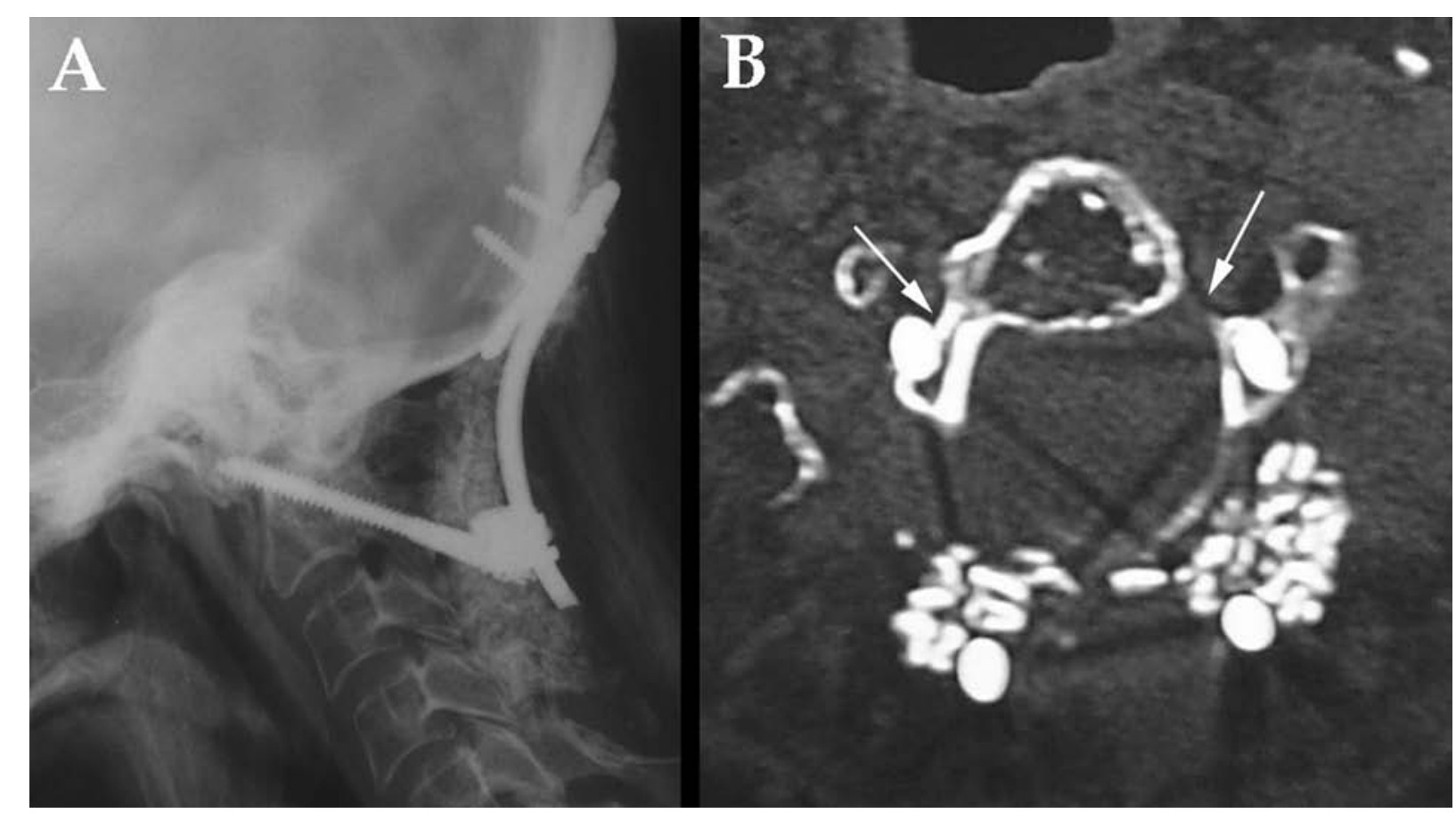

Figura 4. (A) RX lateral postoperatoria. Se observa la correcta orientación del los tornillos C1-C2 hacia el arco anterior del atlas sin perforar su cortical anterior. Los tornillos occipitales, han penetrado en el espacio intracraneal. (B) TAC postoperatorio con ventana para hueso a nivel del axis. Se observan ambos surcos de las arterias vertebrales (flechas) y el trayecto de los tornillos transarticulares por la pars interarticularis del axis. En este caso, los surcos son profundo $(8 \mathrm{~mm}$ del lado izquierdo y $9 \mathrm{~mm}$ del lado derecho) y la pars supera en sólo $2 \mathrm{~mm}$ el diámetro del tornillo en el lado derecho y en $3 \mathrm{~mm}$ en el lado izquierdo. El surco derecho ha sido violado.

chos laminares, alambres o tornillos laminares ${ }^{10,14,16,19}$. Además, los ganchos sublaminares y los cables pueden comprimir la médula cervical, especialmente si existe patología degenerativa acompañante o un canal estrecho congénito ${ }^{8,10}$. Estas técnicas suelen requerir la inclusión de múltiples niveles cervicales.

Una desventaja de la barra occipito-cervical del sistema Olerud $\AA$ (Anatomica, Gothenburg, Sweden) es que no permite realizar una craniectomía suboccipital amplia (Figura 3D). La escama occipital muestra mayor espesor hacia los límites del foramen mágnum y en la línea media, con menor espesor lateralmente. Por este motivo anatómico, la instrumentación en la escama occipital suele dirigirse hacia la línea media. Por otro lado, es en la línea media donde existe mayor riesgo de lesionar estructuras venosas y donde es mayor la necesidad de descompresión cuando está indicado. Se han utilizado para este fin tornillos unicorticales y bicorticales. Estos últimos tienen más riesgo de complicaciones pero son más estables ${ }^{10}$. Existen otras alternativas de atornillado occipital, desde el espacio epidural hacia fuera, aún no bien evaluadas ${ }^{17,21}$. En nuestro caso, la longitud de los tornillos occipitales no parece la adecuada (Figura 4) a pesar de que utilizamos los más cortos dentro de los disponibles.

\section{Bibliografía}

1. Abou, M.A., Solanki, G., Casey, A.T., Crockard, H. A.: Variation of the groove in the axis vertebra for the vertebral artery. Implications for instrumentation. J Bone Joint Surg Br 1997; 79: 820-823.

2. Florensa, R., Noboa, R., Muñoz J., Colet S., Cladellas J.M., Rodríguez M.A., Ley A.: Resultados de la fijación transarticular C1-C2 en una serie de 20 pacientes. Neurocirugia 2002; 13: 429-436.

3. Goel, A., Laheri, V.: Re: Harms J, Melcher P. Posterior $\mathrm{C} 1-\mathrm{C} 2$ fusion with polyaxial screw and rod fixation. Spine 2002; 27: 1589-1590.

4. Goel, A., Laheri, V.: Plate and screw fixation for Atlanto-Axial Subluxation. Acta Neurochirurgica 1994; 129: 47-53.

5. Grob, D., Jeanneret, B., Aebi, M., Markwalder, T. M.: Atlanto-axial fusion with transarticular screw fixation. J Bone Joint Surg Br 1991; 73: 972-976.

6. Harms, J., Melcher, R.P.: Posterior C1-C2 fusion with polyaxial screw and rod fixation. Spine 2001; 26: 2467-2471.

7. Herz, T., Franz, A., Giacomuzzi, S.M., Bale, R., Krismer, M.: Accuracy of spinal navigation for magerl screws. 
Clin Orthop Relat Res 2003; 124-130.

8. Hurlbert, R.J., Crawford, N.R., Choi, W.G., Dickman, C.A.: A biomechanical evaluation of occipitocervical instrumentation: screw compared with wire fixation. J Neurosurg 1999; 90: 84-90.

9. Igarashi, T., Kikuchi, S., Sato, K., Kayama, S., Otani, $\mathrm{K} .:$ Anatomic study of the axis for surgical planning of transarticular screw fixation. Clin Orthop Relat Res 2003; 162-166.

10. Inamasu, J., Kim, D.H., Klugh, A.: Posterior instrumentation surgery for craniocervical junction instabilities: an update. Neurol Med Chir (Tokyo) 2005; 45: 439-447.

11. Kyle, R.A., Rajkumar, S.V.: Multiple myeloma. N Engl J Med 2004; 351: 1860-1873.

12. Malcolm, G.P., Ransford, A.O., Crockard, H.A.: Treatment of non-rheumatoid occipitocervical instability. Internal fixation with the Hartshill-Ransford loop. J Bone Joint Surg Br 1994; 76: 357-366.

13. Martín-Ferrer, S.: Traumatismos de la columna cervical alta: clasificación tipológica, indicaciones terapéuticas y abordajes quirúrgicos. Neurocirugia 2006; 17 : 391-419.

14. Menéndez, J.A., Wright, N.M.: Techniques of posterior C1-C2 stabilization. Neurosurgery 2007; 60: S103S111.

15. Menezes, A.H.: Abnormalities of the craniocervical junction. En R. G. Fessler and L. Sekhar (ed). Atlas of Neurosurgical Techniques. Spine and Peripheral Nerves. New York; Thieme, 2006; pp. 3-11.

16. Oda, I., Abumi, K., Sell, L.C. et al.: Biomechanical evaluation of five different occipito-atlanto-axial fixation techniques. Spine 1999; 24: 2377-2382.

17. Pait, T.G., Al Mefty, O., Boop, F.A. et al.: Insideoutside technique for posterior occipitocervical spine instrumentation and stabilization: preliminary results. J Neurosurg 1999; 90: 1-7.

18. Paramore, C.G., Dickman, C.A., Sonntag, V.K.: The anatomical suitability of the C1-2 complex for transarticular screw fixation. J Neurosurg 1996; 85: 221-224.

19. Puttlitz, C.M., Melcher, R.P., Kleinstueck, F.S. et al.: Stability analysis of craniovertebral junction fixation techniques. J Bone Joint Surg Am 2004; 86-A: 561-568.

20. Rajkumar, S.V., Dispenzieri, A., Kyle, R.A.: Monoclonal gammopathy of undetermined significance, Waldenstrom macroglobulinemia, AL amyloidosis, and related plasma cell disorders: diagnosis and treatment. Mayo Clin Proc 2006; 81: 693-703.

21. Sandhu, F.A., Pait, T.G., Benzel, E., Henderson, F.C.: Occipitocervical fusion for rheumatoid arthritis using the inside-outside stabilization technique. Spine 2003; 28: 414-419.

22. Urculo-Bareño E., Alfaro-Baca R., Astudillo-Alarcon E., Navajas-Cadenal B., Sánchez-Camino A., FigueroaPedrosa M.: Descompresión transoral del tronco cerebral en una impresión basilar secundaria a artritis reumatoidea. Descripción de un caso y revisión de la bibliografía. Rev Neurol 2003; 36: 846-851.

23. Vale, F.L., Oliver, M., Cahill, D.W.: Rigid occipitocervical fusion. J Neurosurg 1999; 91: 144-150.

24. Wright, N.M.: Posterior C2 fixation using bilateral, crossing C2 laminar screws: case series and technical note. J Spinal Disord Tech 2004; 17: 158-162.

25. Wright, N.M., Lauryssen, C.: Vertebral artery injury in C1-2 transarticular screw fixation: results of a survey of the AANS/CNS section on disorders of the spine and peripheral nerves. American Association of Neurological Surgeons/Congress of Neurological Surgeons. J Neurosurg 1998; 88: 634-640.

Samprón, N.; Arrazola, M.; Urculo E.: Plasmocitoma de base craneal con inestabilidad cráneo-cervical. Neurocirugía 2009; 20: 478-483.

Correspondencia postal: Nicolás Samprón. Servicio de Neurocirugía. Hospital Donostia. Paseo Dr. Beguiristain s/n. 20080 San Sebastián. España. 